\title{
ANTIMICROBIAL SUSCEPTIBILITY OF BACILLUS ANTHRACIS STRAINS FROM HUNGARY
}

\author{
Zsuzsa KREIZINGeR ${ }^{1}$, Kinga Mária SUlYOK ${ }^{1}$, László MAKRAI ${ }^{2}$, Zsuzsanna RÓNAI ${ }^{3}$, \\ László FODOR ${ }^{2}$, Szilárd JÁNOSI ${ }^{3}$ and Miklós GYURANECZ ${ }^{1 *}$ \\ ${ }^{1}$ Institute for Veterinary Medical Research, Centre for Agricultural Research, \\ Hungarian Academy of Sciences, Hungária krt. 21, H-1143 Budapest, Hungary; \\ ${ }^{2}$ Department of Microbiology and Infectious Diseases, Faculty of Veterinary Science, \\ Szent István University, Budapest, Hungary; ${ }^{3}$ Veterinary Diagnostic Directorate, \\ National Food Chain Safety Office, Budapest, Hungary
}

(Received 7 October 2015; accepted 28 October 2015)

The susceptibility of 29 Bacillus anthracis strains, collected in Hungary between 1933 and 2014, was tested to 10 antibiotics with commercially available minimum inhibitory concentration (MIC) test strips. All strains were susceptible to amoxicillin, ciprofloxacin, clindamycin, doxycycline, gentamicin, penicillin, rifampicin, and vancomycin. Intermediate susceptibility to erythromycin and cefotaxime was detected in $17.2 \%(5 / 29)$ and $58.6 \%$ (17/29) of the strains, respectively. Correlations were not observed between the isolation date, location, host species, genotype, and antibiotic susceptibility profile of strains.

Key words: Antibiotic susceptibility, anthrax, Bacillus anthracis, Hungary

Bacillus anthracis, the causative agent of anthrax, is a spore-forming, Gram-positive, zoonotic bacterium, a potential biological warfare agent which primarily infects herbivores, but can cause serious disease in humans with cutaneous, gastrointestinal, pulmonary, and injectional forms (WHO, 2008; Fasanella et al., 2010; Hanczaruk et al., 2014). The extremely resistant spore is able to survive in the soil for decades (Fasanella et al., 2010). Bacillus anthracis is susceptible to most antibiotics used in therapy (Lightfoot et al., 1990; Doğanay and Aydin, 1991; Odendaal et al., 1991; Cavallo et al., 2002; Coker et al., 2002; Mohammed et al., 2002; Turnbull et al., 2004; Luna et al., 2007; Caplan et al., 2009; Habrun et al., 2011; Ortatatli et al., 2012; Quinn et al., 2011). The main recommended antibiotics to treat human anthrax cases are ciprofloxacin and doxycycline, apart from penicillin (WHO, 2008). Animals showing clinical signs of anthrax are also treated with different antibiotics, mainly penicillin and oxytetracycline (Quinn et al., 2011).

\footnotetext{
*Corresponding author; E-mail: m.gyuranecz@gmail.com; Phone: 0036 (1) 467-4060;
} Fax: 0036 (1) 467-4076 


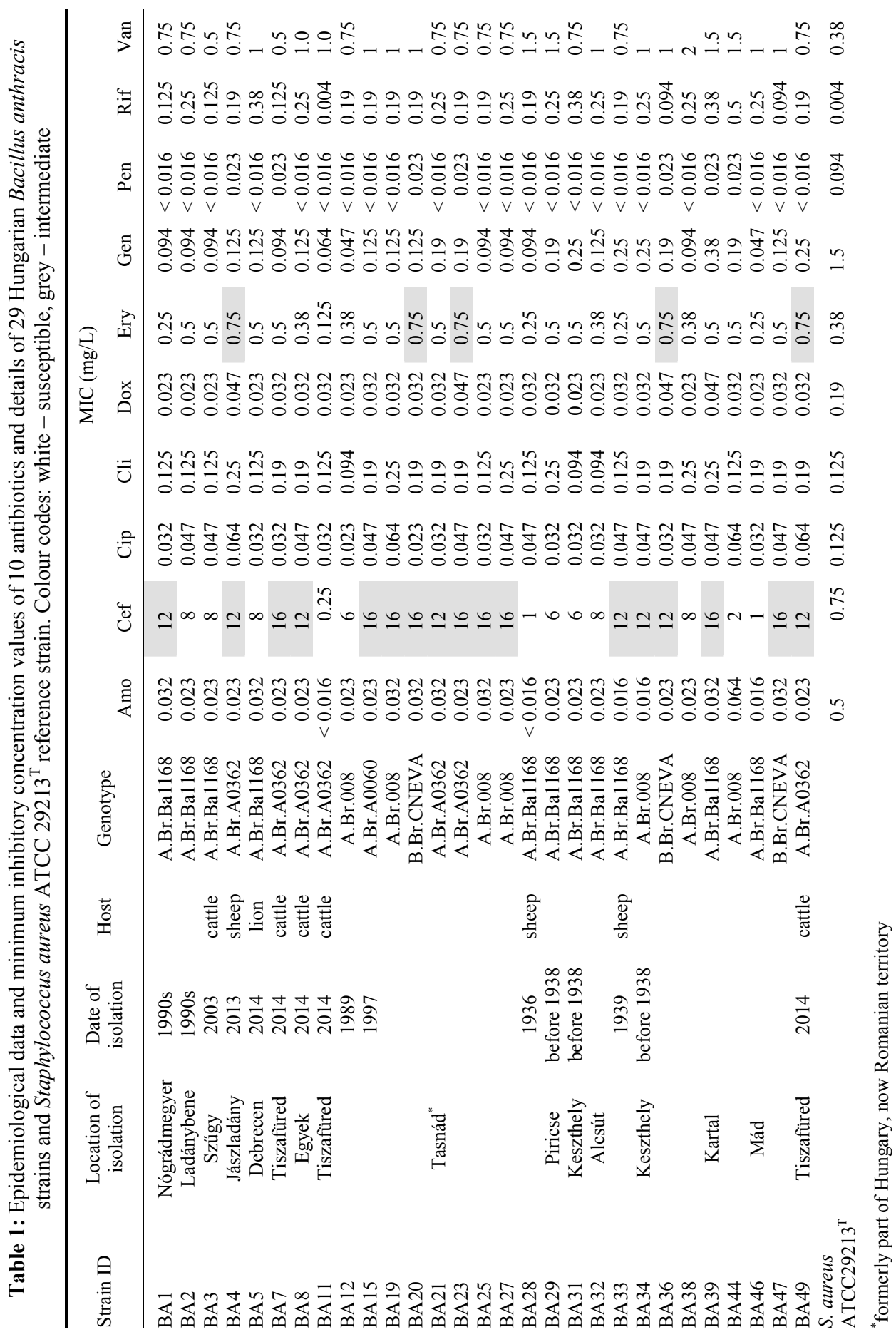


Commercially available minimum inhibitory concentration (MIC) test strips (Etest, BioMérieux Inc., Marcy l'Etoile, France) proved to be a simple and flexible method to determine the antibiotic susceptibility profiles with MIC values of $B$. anthracis strains. The effectiveness of the strips is comparable to that of conventional methods used for MIC value determination (Turnbull et al., 2004; Luna et al., 2007).

The aim of this study was to determine the susceptibility of $B$. anthracis strains from Hungary to 10 antibiotics with commercially available MIC test strips.

\section{Materials and methods}

The study involved $29 \mathrm{~B}$. anthracis strains isolated from diverse host species in various parts of Hungary between 1933 and 2014 (Table 1). The strains were identified and genotyped as described previously. Briefly, the strains were identified on species level with a dual-probe TaqMan assay targeting a single nucleotide polymorphism (SNP) in the plcR gene (Easterday et al., 2005). The genotypes of the isolates were determined with melt mismatch amplification mutation assays according to Birdsell et al. (2012) targeting the SNPs identified by Van Ert et al. (2007).

The susceptibility of the strains to 10 antimicrobial agents (amoxicillin, cefotaxime, ciprofloxacin, clindamycin, doxycycline, erythromycin, gentamicin, penicillin, rifampicin, and vancomycin) was determined with commercially available MIC test strips (Etest, BioMérieux Inc., Marcy l'Etoile, France). Staphylococcus aureus ATCC $29213^{\mathrm{T}}$ served as a positive control. The strains were cultured on Mueller-Hinton agar plates overnight at $37^{\circ} \mathrm{C}$. Colony suspensions in $3 \mathrm{ml}$ of physiological saline, equalled to a density of $0.5 \mathrm{McF}$ arland, were consistently taken on $5 \mathrm{~mm}$-thick Mueller-Hinton agar with sterile swab. An E-test strip was placed on each plate after approximately $10 \mathrm{~min}$, to allow absorption of excess moisture into the agar. The plates were incubated at $37{ }^{\circ} \mathrm{C}$ for $20 \mathrm{~h}$ and MIC values were read according to the manufacturer's instructions. We interpreted the breakpoints according to Clinical and Laboratory Standards Institute (CLSI, 2011) standards for B. anthracis and CLSI standards for Staphylococcus sp. where standards were unavailable for B. anthracis (Table 2). All B. anthracis manipulations were performed in a biosafety level 3 laboratory using class III biosafety cabinet.

\section{Results}

Based on the $\mathrm{MIC}_{90}$ values all Hungarian B. anthracis isolates were susceptible to penicillin $(0.023 \mathrm{mg} / \mathrm{L})$, amoxicillin $(0.032 \mathrm{mg} / \mathrm{L})$, ciprofloxacin $(0.064 \mathrm{mg} / \mathrm{L})$, doxycycline $(0.047 \mathrm{mg} / \mathrm{L})$, rifampicin $(0.38 \mathrm{mg} / \mathrm{L})$, vancomycin 
$(1.5 \mathrm{mg} / \mathrm{L})$, gentamicin $(0.25 \mathrm{mg} / \mathrm{L})$, and clindamycin $(0.25 \mathrm{mg} / \mathrm{L})$ (Tables 1 and 2$)$. Most of the strains $(24 / 29,82.8 \%)$ were susceptible to erythromycin, but intermediately susceptible strains $(5 / 29,17.2 \%)$ also occurred. The strains were susceptible $(12 / 29,41.4 \%)$ or intermediately $(17 / 29,58.6 \%)$ susceptible to cefotaxime.

Although background information about the tested B. anthracis strains was limited, correlations were not observed between the isolation date, location, host species, genotype, and antibiotic susceptibility profile of the $29 \mathrm{~B}$. anthracis strains (Table 1).

Table 2

Susceptibility profile of 29 Bacillus anthracis strains for 10 antimicrobial agents

\begin{tabular}{|c|c|c|c|c|c|c|c|c|}
\hline \multirow{2}{*}{ Antibiotic } & \multicolumn{3}{|c|}{$\mathrm{MIC}(\mathrm{mg} / \mathrm{L})$} & \multicolumn{2}{|c|}{ Breakpoints } & \multicolumn{3}{|c|}{$\%$ of isolates } \\
\hline & Range & $50 \%$ & $90 \%$ & $\mathrm{~S}(\leq)$ & $\mathrm{R}(\geq)$ & $\mathrm{S}$ & I & $\mathrm{R}$ \\
\hline Amoxicillin & $0.016-0.064$ & 0.023 & 0.032 & 0.25 & $0.5^{\mathrm{b}}$ & 100 & & \\
\hline Cefotaxime & $0.25-16$ & 12 & 16 & 8 & $64^{b}$ & 41.4 & 58.6 & \\
\hline Ciprofloxacin & $0.023-0.064$ & 0.047 & 0.064 & 0.5 & $\mathrm{NA}^{\mathrm{a}}$ & 100 & & \\
\hline Clindamycin & $0.094-0.25$ & 0.19 & 0.25 & 0.5 & $4^{b}$ & 100 & & \\
\hline Doxycycline & $0.023-0.047$ & 0.032 & 0.047 & 1.0 & $\mathrm{NA}^{\mathrm{a}}$ & 100 & & \\
\hline Erythromycin & $0.125-0.75$ & 0.5 & 0.75 & 0.5 & $8^{\mathrm{b}}$ & 82.8 & 17.2 & \\
\hline Gentamicin & $0.047-0.38$ & 0.125 & 0.25 & 4 & $16^{\mathrm{b}}$ & 100 & & \\
\hline Penicillin & $<0.016-0.023$ & $<0.016$ & 0.023 & 0.12 & $0.25^{\mathrm{a}}$ & 100 & & \\
\hline Rifampicin & $0.004-0.5$ & 0.19 & 0.38 & 1 & $4^{b}$ & 100 & & \\
\hline Vancomycin & $0.5-2$ & 1 & 1.5 & 4 & $32^{b}$ & 100 & & \\
\hline
\end{tabular}

MIC: minimum inhibitory concentration; NA: data not available; S: susceptible; I: intermediate; R: resistant; ${ }^{\mathrm{a} C L S I}$ standard breakpoints for B. anthracis; ${ }^{\mathrm{b}}$ CLSI standard breakpoints for Staphylococcus $\mathrm{sp}$.

\section{Discussion}

The objective of this work was to study the antibiotic susceptibility profile of $B$. anthracis strains from Hungary. Eight of the ten examined antibiotics also appear on the list of antibiotics recommended for human anthrax treatment (WHO, 2008).

Penicillin $\mathrm{G}$ is the primary recommended antibiotic to treat cutaneous anthrax cases without complications. Amoxicillin can be an alternative to penicillin (WHO, 2008). Penicillin and amoxicillin resistance was confirmed in several studies, within a range of $1-12 \%$ of the strains examined (Lightfoot et al., 1990; Cavallo et al., 2002; Coker et al., 2002; Mohammed et al., 2002; Turnbull et al., 2004). All Hungarian strains, however, proved to be highly susceptible to penicillin and amoxicillin.

Erythromycin is also a possible alternative in case of penicillin allergy (WHO, 2008). Moderately sensitive strains appeared in several studies (Cavallo et al., 2002; Mohammed et al., 2002; Luna et al., 2007; Ortatatli et al., 2012), and 
two resistant strains were described in Turkey (Ortatatli et al., 2012). In the current study, $17.2 \%$ of the Hungarian strains were only intermediately sensitive to erythromycin. These data suggest that erythromycin should be avoided in anthrax treatment in Hungary.

Ciprofloxacin and doxycycline are recommended in life-threatening cases and in case of penicillin allergy (WHO, 2008). Ciprofloxacin resistance has not been described yet. Resistance against doxycycline was observed in one strain (MIC $4 \mathrm{mg} / \mathrm{L}$ ) in Turkey (Ortatatli et al., 2012). All examined strains from Hungary were susceptible to both antibiotics.

Rifampicin is recommended as complementary treatment in anthrax meningoencephalitis, clindamycin and vancomycin in inhalation anthrax, and gentamicin in gastrointestinal anthrax (WHO, 2008). Rifampicin, vancomycin and clindamycin resistance has not been reported yet. Gentamicin resistance was observed in two cases (MIC $=32 \mathrm{mg} / \mathrm{L}$ ) in Turkey (Ortatatli et al., 2012). All Hungarian strains proved to be susceptible to rifampicin, vancomycin, clindamycin, and gentamicin as well.

Most $B$. anthracis stains are resistant or intermediately susceptible to cefotaxime according to previous findings (Doğonay and Aydin, 1991; Odendaal et al., 1991; Turnbull et al., 2004; Habrun et al., 2011; Ortatatli et al., 2012). Turnbull et al. (2004) found one susceptible strain (MIC $3 \mathrm{mg} / \mathrm{L}$ ) with the Etest method, while Doğanay and Aydin (1991) described five susceptible strains with the disc diffusion test. In the present study, $41.4 \%$ of the Hungarian strains were susceptible to cefotaxime. Additionally, no resistant strains were identified. The $\mathrm{MIC}_{90}$ values proved to be lower in our study compared to other studies (Doğanay and Aydin, 1991; Odendaal et al., 1991; Turnbull et al., 2004; Habrun et al., 2011; Ortatatli et al., 2012).

In conclusion, despite the temporal, geographic, host and genetic diversity of the tested $B$. anthracis isolates, their susceptibility profiles were highly similar. According to the present study, penicillin, amoxicillin, ciprofloxacin and doxycycline are the primary choices to treat anthrax, but gentamicin, vancomycin, rifampicin and clindamycin could also be used against $B$. anthracis in Hungary. The application of erythromycin and cefotaxime should be avoided in the treatment of anthrax.

\section{Acknowledgements}

The study was supported by the Lendület (Momentum) programme (LP2012-22) of the Hungarian Academy of Sciences. We thank Veronika Hrivnák and Alexandra Pásztor for their technical assistance. 


\section{References}

Birdsell, D. N., Pearson, T., Price, E. P., Hornstra, H. M., Nera, R. D., Stone, N., Gruendike, J., Kaufman, E. L., Pettus, A. H., Hurbon, A. N., Buchhagen, J. L., Harms, N. J., Chanturia, G., Gyuranecz, M., Wagner, D. M. and Keim, P. S. (2012): Melt analysis of mismatch amplification mutation assays (Melt-MAMA): a functional study of a cost-effective SNP genotyping assay in bacterial models. PLoS One 7, e32866.

Caplan, D. M., Ivana, S. and Caplan, M. E. (2009): Susceptibility to antibiotics of Bacillus anthracis strains isolated in Romania. Roum. Arch. Microbiol. Immunol. 68, 106-110.

Cavallo, J. D., Ramisse, F., Girardet, M., Vaissaire, J., Mock, M. and Hernandez, E. (2002): Antibiotic susceptibilities of 96 isolates of Bacillus anthracis isolated in France between 1994 and 2000. Antimicrob. Agents Chemother. 46, 2307-2309.

Clinical and Laboratory Standards Institute (2011): Performance Standards for Antimicrobial Susceptibility Testing: Thirtieth Informational Supplement M45-A2. CLSI, Wayne, PA, USA.

Coker, P. R., Smith, K. L. and Hugh-Jones, M. E. (2002): Antimicrobial susceptibilities of diverse Bacillus anthracis isolates. Antimicrob. Agents Chemother. 46, 3843-3845.

Doğanay, M. and Aydin, N. (1991): Antimicrobial susceptibility of Bacillus anthracis. Scand. J. Infect. Dis. 23, 333-335.

Easterday, W. R., Van Ert, M. N., Simonson, T. S., Wagner, D. M., Kenefic, L. J., Allender, C. J. and Keim, P. (2005): Use of single nucleotide polymorphisms in the plcR gene for specific identification of Bacillus anthracis. J. Clin. Microbiol. 43, 1995-1997.

Fasanella, A., Galante, D., Garofolo, G. and Jones, M. H. (2010): Anthrax undervalued zoonosis. Vet. Microbiol. 140, 318-331.

Habrun, B., Racic, I., Kompes, G., Spicic, S., Benic, M., Mihaljevic, Z. and Cvetnic, Z. (2011): The antimicrobial susceptibility and virulence factors of Bacillus anthracis strains isolated in Croatia. Veterinarni Medicina 56, 22-27.

Hanczaruk, M., Reischl, U., Holzmann, T., Frangoulidis, D., Wagner, D. M., Keim, P. S., Antwerpen, M. H., Meyer, H. and Grass, G. (2014): Injectional anthrax in heroin users, Europe, 2000-2012. Emerg. Infect. Dis. 20, 322-323.

Lightfoot, N. F., Scott, R. J. D. and Turnbull, P. C. B. (1990): Antimicrobial susceptibility of Bacillus anthracis. Salisbury Med. Bull. 68, 95-98.

Luna, V. A., King, D. S., Gulledge, J., Cannons, A. C., Amuso, P. T. and Cattani, J. (2007): Susceptibility of Bacillus anthracis, Bacillus cereus, Bacillus mycoides, Bacillus pseudomycoides and Bacillus thuringiensis to 24 antimicrobials using Sensititre automated microbroth dilution and Etest agar gradient diffusion methods. J. Antimicrob. Chemother. 60, 555-567.

Mohammed, M. J., Marston, C. K., Popovic, T., Weyant, R. S. and Tenover, F. C. (2002): Antimicrobial susceptibility testing of Bacillus anthracis: comparison of results obtained by using the National Committee for Clinical Laboratory Standards broth microdilution reference and Etest agar gradient diffusion methods. J. Clin. Microbiol. 40, 1902-1907.

Odendaal, M. W., Pieterson, P. M., de Vos, V. and Botha, A. D. (1991): The antibiotic sensitivity patterns of Bacillus anthracis isolated from the Kruger National Park. Onderstepoort J. Vet. Res. 58, 17-19.

Ortatatli, M., Karagoz, A., Percin, D., Kenar, L., Kilic, S. and Durmaz, R. (2012): Antimicrobial susceptibility and molecular subtyping of 55 Turkish Bacillus anthracis strains using 25loci multiple-locus VNTR analysis. Comp. Immunol. Microbiol. Infect. Dis. 35, 355-361.

Quinn, P. J., Markey, B. K., Leonard, F. C., Hartigan, P., Fanning, S. and FitzPatrick, E. S. (2011): Veterinary Microbiology and Microbial Disease. 2nd edition. Blackwell, Oxford.

Turnbull, P. C., Sirianni, N. M., LeBron, C. I., Samaan, M. N., Sutton, F. N., Reyes, A. E. and Peruski, L. F. Jr. (2004): MICs of selected antibiotics for Bacillus anthracis, Bacillus cereus, 
Bacillus thuringiensis, and Bacillus mycoides from a range of clinical and environmental sources as determined by the Etest. J. Clin. Microbiol. 42, 3626-3634.

Van Ert, M. N., Easterday, W. R., Huynh, L. Y., Okinaka, R. T., Hugh-Jones, M. E., Ravel, J., Zanecki, S. R., Pearson, T., Simonson, T. S., U'Ren, J. M., Kachur, S. M., LeademDougherty, R. R., Rhoton, S. D., Zinser, G., Farlow, J., Coker, P. R., Smith, K. L., Wang, B., Kenefic, L. J., Fraser-Liggett, C. M., Wagner, D. M. and Keim, P. (2007): Global genetic population structure of Bacillus anthracis. PloS One 2, e461.

World Health Organization and International Office of Epizootics (2008): Anthrax in humans and animals. WHO, Geneva, Switzerland. 